\title{
Numerical simulation and experimental verification of gas streams feeding a gliding discharge plasma reactor *
}

\author{
Teresa Opalińska ${ }^{1, a}$, Małgorzata Majdak ${ }^{1}$, Janusz Piechna ${ }^{2}$, Witold Selerowicz ${ }^{2}$, and Bartłomiej Wnek ${ }^{1}$ \\ ${ }^{1}$ Tele and Radio Research Institute, 44/50 Długa str., 00-241 Warsaw, Poland \\ ${ }^{2}$ Warsaw University of Technology, The Faculty of Power and Aeronautical Engineering, \\ 24 Nowowiejska str., 00-665 Warsaw, Poland
}

Received: 1 October 2012 / Received in final form: 5 December 2012 / Accepted: 10 December 2012 Published online: 15 February 2013 - (c) The Author(s) 2013

\begin{abstract}
The modular device for the waste utilization, using the pyrolytic-plasma method, consists of, among others, the plasma reactor operating on the gliding discharge principles. The reactor is applied to oxidize hydrocarbons created in the process of the waste pyrolysis. Thus, the plasma reactor operation influences significantly the qualitative and quantitative composition of the output gases. Finding the most advantageous construction of the plasma reactor and the process parameters ensures full and complete oxidation of hydrocarbons. In the waste utilization process, two streams of gases were introduced into the plasma reactor through the system of two coaxial nozzles. The first stream was a mixture of argon and hydrocarbons and the second one was oxygen. Two different methods of gas introduction were considered. Methane was used as a model hydrocarbon both in calculations and in the experiments. On the basis of numerical calculations (Fluent commercial program), it was found that the most advantageous method of gas introduction into the reactor was the one in which the mixture of argon and hydrocarbons was introduced through the central nozzle and the oxygen was introduced through the outer one. This conclusion was confirmed experimentally.
\end{abstract}

\section{Introduction}

A nonequilibrium plasma is generated at the atmospheric pressure in the gliding discharge supplied from a DC or $\mathrm{AC}$ source. The plasma is characterized by the electron energy of about $1 \mathrm{eV}$ and the neutral gas temperature of 1500-2000 K, depending on the power applied [1,2]. The properties of the generated plasma and a relatively easy way of the discharge generation cause that a practical application for this discharge is often investigated [3-6]. The gliding discharge is applied, among others, in a plasma reactor, which may be a part of the waste utilization device. The utilization process in this device consists of: (1) the pyrolysis of waste without the air access; (2) the oxidation of the pyrolysis products in gliding discharge and (3) the purification of the process gases [7].

The oxidation process has a cardinal influence on the quality of gases leaving the device. For this the plasma reactor should enable the efficient oxidation of hydrocarbons, which are the main products of the waste pyrolysis. The highest efficiency of reaction will be obtained if all

\footnotetext{
* Contribution to the Topical Issue "13th International Symposium on High Pressure Low Temperature Plasma Chemistry (Hakone XIII)", Edited by Nicolas Gherardi, Henryca Danuta Stryczewska and Yvan Ségui.

a e-mail: tzopalinska@gmail.com
}

reactants are introduced into the zone of discharge. Diverse solutions of the reactor construction are proposed to improve the efficiency of its chemical reaction. It was found that this efficiency can be better if the circulation of reagents inside the volume of reactor is used, and then the mixture of reacting gases flows repeatedly through the zone of discharge. To force the gases circulation, pump is installed outside the reactor [8]. Similar effect can be obtained in the reactor, in which a system of, e.g., three electrodes is placed repeatedly along the reactor tube at a specified distance. Gases flow through several zones of discharge generated between electrodes [9]. In another reactor, the stream of gases flows along the electrodes which are positioned in a channel expanded in the direction of the gas flow. The walls of the channel reduce the gas stream to the area of discharge [10]. Apart from different reactor constructions, various arrangements of electrodes are used in order to enlarge the area of discharge what should increase the reaction efficiency. Rotary electrodes would be used to increase the discharge length [11]. A wire electrode with a reverse vortex flow makes the zone of discharge expand to the total reactor volume [12]. The authors selected the simplest way of enlarging the volume of discharge, using three-electrode reactor powered by 3 -phase alternating current. An important structural element of such a reactor used in the study is the system 

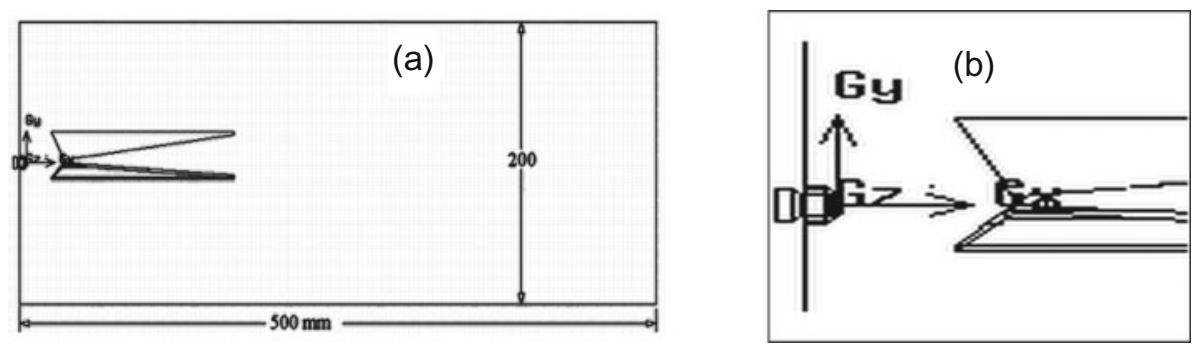

Fig. 1. (a) The scheme of plasma reactor and (b) enlarged part of reactor comprising the system of nozzles.

feeding the reactants to the plasma rector. The system consists of two individually designed coaxial nozzles. One of them is used for transporting the pyrolysis gases by means of the argon agent into the plasma reactor. The second nozzle is used to add up oxygen. It is very important that both streams are exactly mixed just before they enter the zone of electric discharge just to obtain the stoichiometric mixture. The resultant stream of mixed gases should have a shape enabling the generation of discharge in the whole stream volume. Thus, all reagents can completely react. The reagents, which flow outside the discharge volume, may not react, because the process occurs at the pressure close to atmospheric. This fact should be taken into account if the practical application of the plasma reactor is considered.

The proper design of nozzles having the correct shape required several theoretical studies and a practical verification $[13,14]$. The aim of this project was to determine the methods of gases introduction into the plasma reactor for the designed nozzle system. It was done by the numerical simulation of gas streams feeding the reactor and then by the experimental verification of the calculation results.

\section{Numerical simulations}

\subsection{Assumptions}

The plasma reactor is a part of apparatus for waste utilization. Its principle of operation can be described as follows. The waste is pyrolyzed in the atmosphere of argon. The hydrocarbons generated in the process are introduced into the plasma reactor in the stream of argon. Oxygen is introduced into the reactor separately. The process of hydrocarbons oxidation takes place in the plasma reactor.

The hypothetical reactor consisted of a reaction chamber in the form of a cylinder of $200 \mathrm{~mm}$ diameter and a length of $500 \mathrm{~mm}$, which was equipped with a set of two coaxial nozzles at one end and was open at the other (Fig. 1). The shape of nozzles facilitated the process of mixing of both gas streams (Fig. 2). The mixed gas stream flowed along the three electrodes, between them.

It was assumed that the $2.0 \mathrm{~kg}$ of waste that containing high amount of elemental carbon was fully pyrolyzed to methane during $1 \mathrm{~h}$ of the process. Methane was mixed with argon and fed into the plasma reactor through one of the two nozzles. Oxygen was introduced through the

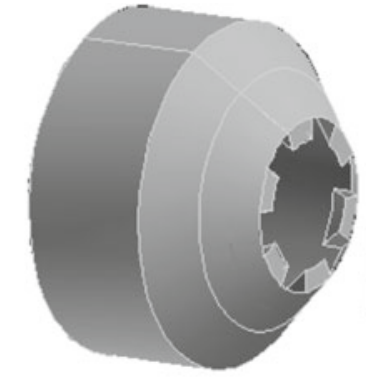

$\rightarrow x$

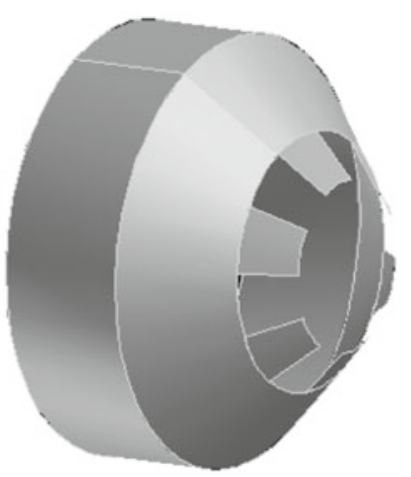

Fig. 2. The system of nozzles applying in calculations.

second nozzle. Calculations were performed for the following mass flow rates: methane $=4.9 \times 10^{-4} \mathrm{~kg} / \mathrm{s}$; argon $=9.8 \times 10^{-4} \mathrm{~kg} / \mathrm{s}$; oxygen $=1.9 \times 10^{-3} \mathrm{~kg} / \mathrm{s}$. The flow rate of oxygen was calculated including the stoichiometry of the reaction of methane oxidation into carbon dioxide:

$$
\mathrm{CH}_{4}+2 \mathrm{O}_{2} \rightarrow \mathrm{CO}_{2}+2 \mathrm{H}_{2} \mathrm{O} \text {. }
$$

The mixture temperature of methane with argon was $1100 \mathrm{~K}$ and the temperature of oxygen was $273 \mathrm{~K}$. The gases leaving the nozzles were mixed in the plasma reactor. The stream of the mixed gases should reach the stoichiometric composition just before the zone of discharge. The shape of the mixed gases stream should facilitate the generation of discharge in the whole volume of the stream.

\subsection{Calculations}

Calculations were performed for two cases. In the first case the constitution of gases in the stream was calculated ignoring the reaction of methane oxidation. Only the process of gas streams mixing was taken into account. In the second case, apart from the streams mixing, the reaction of methane oxidation was considered. For each case two methods of gas introducing into the reactor were taken into account. The first method consisted in introducing into the reactor the mixture of argon and methane through the central nozzle and oxygen through the outer one. In the second method, oxygen was introduced through the central nozzle and the mixture of argon and methane through the outer one (Fig. 3). 
T. Opalińska et al.: Numerical simulation and experimental verification of gas streams

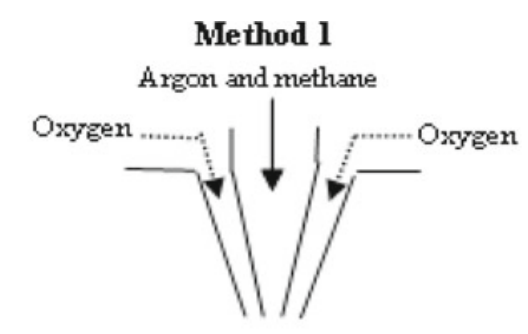

Method 2

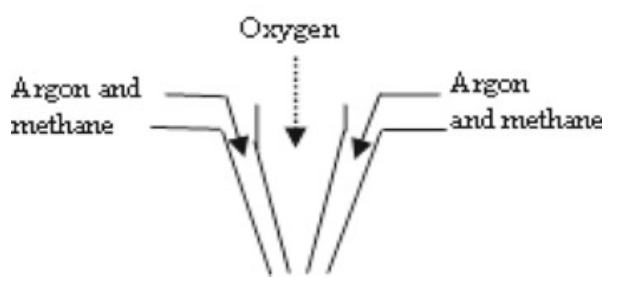

Fig. 3. Methods of gases introduction into the plasma reactor.

A computer simulation of gas streams supplying the plasma reactor was performed using a computational fluid dynamics commercial program (CFC) Fluent. Two simulations of flows mixing were taken into account: (1) excluding the methane oxidation and (2) including the oxidation of methane.

In the domain representing the chamber the following boundary conditions were used: (1) zero gas velocity at the wall, (2) condition representing the outflow to an area of constant pressure, (3) axis condition in axiallysymmetric system. Navier-Stokes equations for steady flow of compressible, viscous, multi-component fluid have been solved using a coupled solver (density-based formulation). We used two models of turbulence: the model of SpalartAllmaras and $k$-epsilon model. The robust S-A model was used at the initial steps of investigation and was later replaced by the $k$-epsilon model required in some simulations of methane oxidation process. The Arrhenius equation was used for calculation of methane oxidation reaction. The heat transferred by radiation was calculated using the Rosseland's model of radiation.

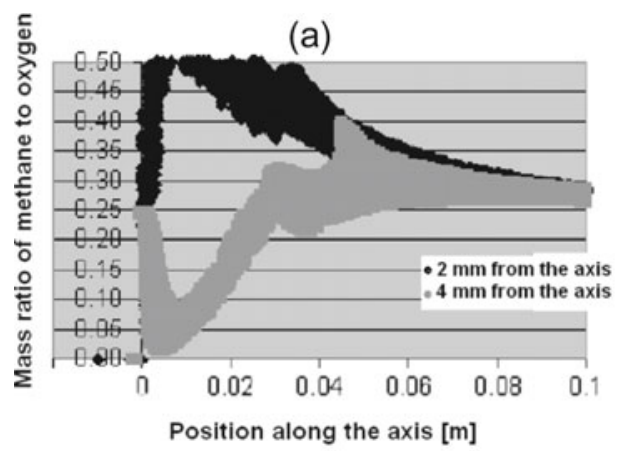

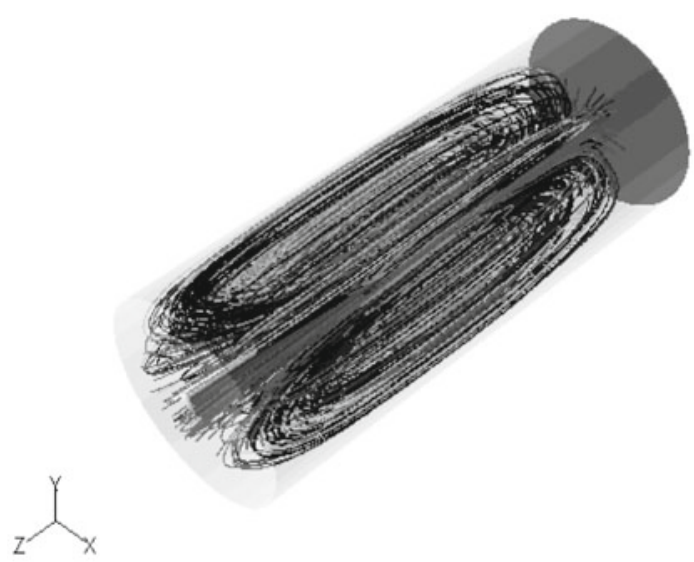

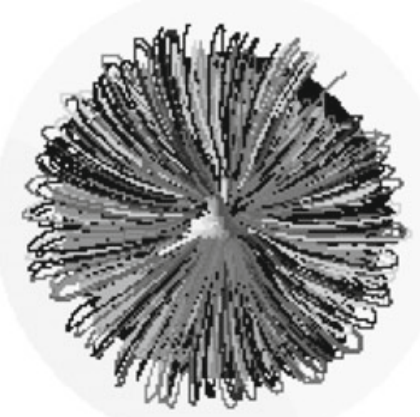

Fig. 4. Runs of streamlines in the rector. Methane oxidation was not considered.

\subsection{Results of calculations}

The runs of streamlines in the reactor chamber (Fig. 4) showed that the gas flow calculated without the oxidation process was stable and regular. For the methane oxidation in the plasma reactor the distribution of the mass ratio of methane to oxygen along the reactor axis plays an important role. The ratio should be 0.26 , because then the composition of gas stream corresponds to the stoichiometric composition of methane and oxygen in the reaction of complete methane oxidation. The distribution of

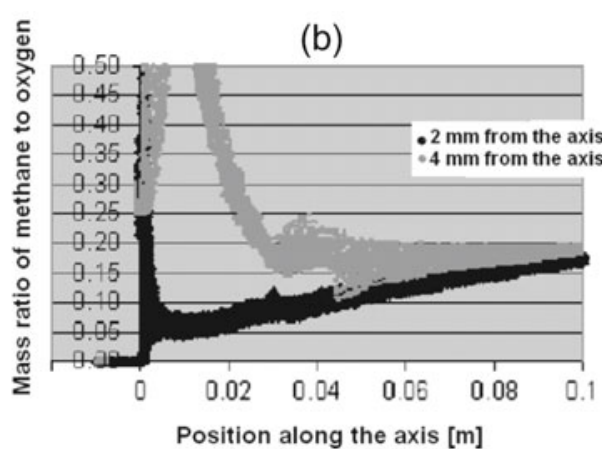

Fig. 5. The variation of mass ratio of methane to oxygen along the axis of reactor calculated numerically in the distance from the axis of reactor of $2 \mathrm{~mm}$ and $4 \mathrm{~mm}$. The methane was introduced into reactor applying: (a) the first method, (b) the second method (methane oxidation was not considered). 
mass ratio of methane to oxygen along the axis of reactor was calculated at a distance of 2 and $4 \mathrm{~mm}$ from the axis. The distance of $2 \mathrm{~mm}$ was selected because it is the smallest distance between the reactor electrodes. The distance of $4 \mathrm{~mm}$ was chosen for calculations because the distance between electrodes increases with the length of electrodes. The calculations showed that the stoichiometric composition of methane and oxygen in the stream of gases was achieved when the mixture of argon and methane was introduced into the reactor through the central nozzle and oxygen through the outer one, irrespective of the distance from the reactor axis (Fig. 5). For both distances the stoichiometric composition of the stream was achieved at a distance of $60 \mathrm{~mm}$ from the face of the nozzles.

In the further calculations the methane oxidation process was taken into account. The distribution of shear stress was more uniform when the methane entered the reactor through the central nozzle (Fig. 6). In such a case the values of shear stress were larger than those for the gases introduced into the reactor using the second method. It means that the mixing of gases was more intensive when the first method was used.

The distributions of the mass fraction of methane and that of oxygen in the stream of gases for both methods of gases introduction into the plasma reactor were calculated and compared (Figs. 7 and 8). For the stoichiometric mixture the mass fraction of methane and that of oxygen were respectively 0.145 and 0.56 (including argon in gas mixture). When the first method of gases introduction was taken into account, the stoichiometric mixture of gases was obtained when the distance between electrodes was the shortest. In that point the oxidation of methane should start. If the second method was used in the calculations, then nearby the place where the discharge started, the methane mass fraction was too small and oxygen fraction was too high in comparison to the stoichiometric mixture.

The results of oxidation can be best assessed by the concentration of methane at the chamber outlet that has not reacted in the process. The largest methane mass fraction was 0.005 when methane was introduced into the reactor by the first method but when the second method was used the mass fraction was 0.008 (Fig. 9).

The results of calculation showed that the first method of feeding methane into the reactor is better than the second one, regardless of whether the methane oxidation is taken into account or not.

\section{Experimental part}

\subsection{Experimental setup}

The verification and the correctness of the conclusions drawn from the calculations required designing and building a plasma reactor of dimensions corresponding to the one used for the calculation. The reactor was equipped with a set of two coaxial nozzles, which was slightly modified in comparison with that shown in Figure 2. The central nozzle was simultaneously a mixing nozzle. It had six
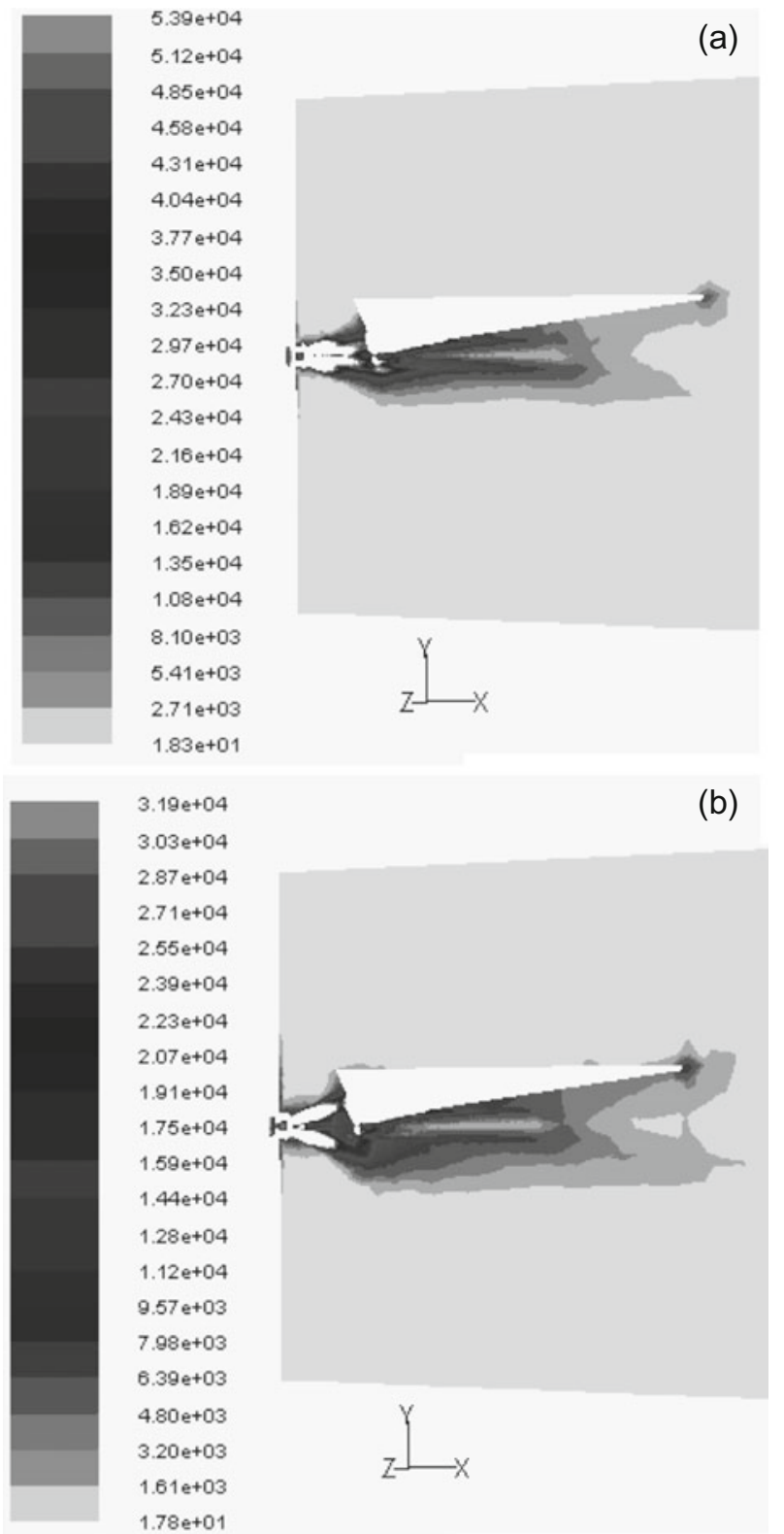

(b)

Fig. 6. The calculated distribution of shear stresses along the stream of gases in the plane of symmetry (methane oxidation was considered). The mixture of methane and argon was introduced into reactor (a) through the central nozzle and (b) through the outer one.

slots breaking the gas stream into six smaller jets that facilitated the process of mixing. The outer nozzle was plain. The reactor had three knife-shaped electrodes of the size and shape selected on the basis of numerical calculations and was supplied with a high voltage $(1.4 \mathrm{kV}) 50 \mathrm{~Hz} \mathrm{AC}$ source. The reactor supply power was calculated on the basis of electric energy measured on the transformer primary winding supplying the reactor.

The reactor was surrounded with the systems of argon, methane and oxygen feeding. The gas input flow was measured with the Bronkhorst mass flow meters, and the post-reaction gas flow was measured with a gas meter. 
T. Opalińska et al.: Numerical simulation and experimental verification of gas streams

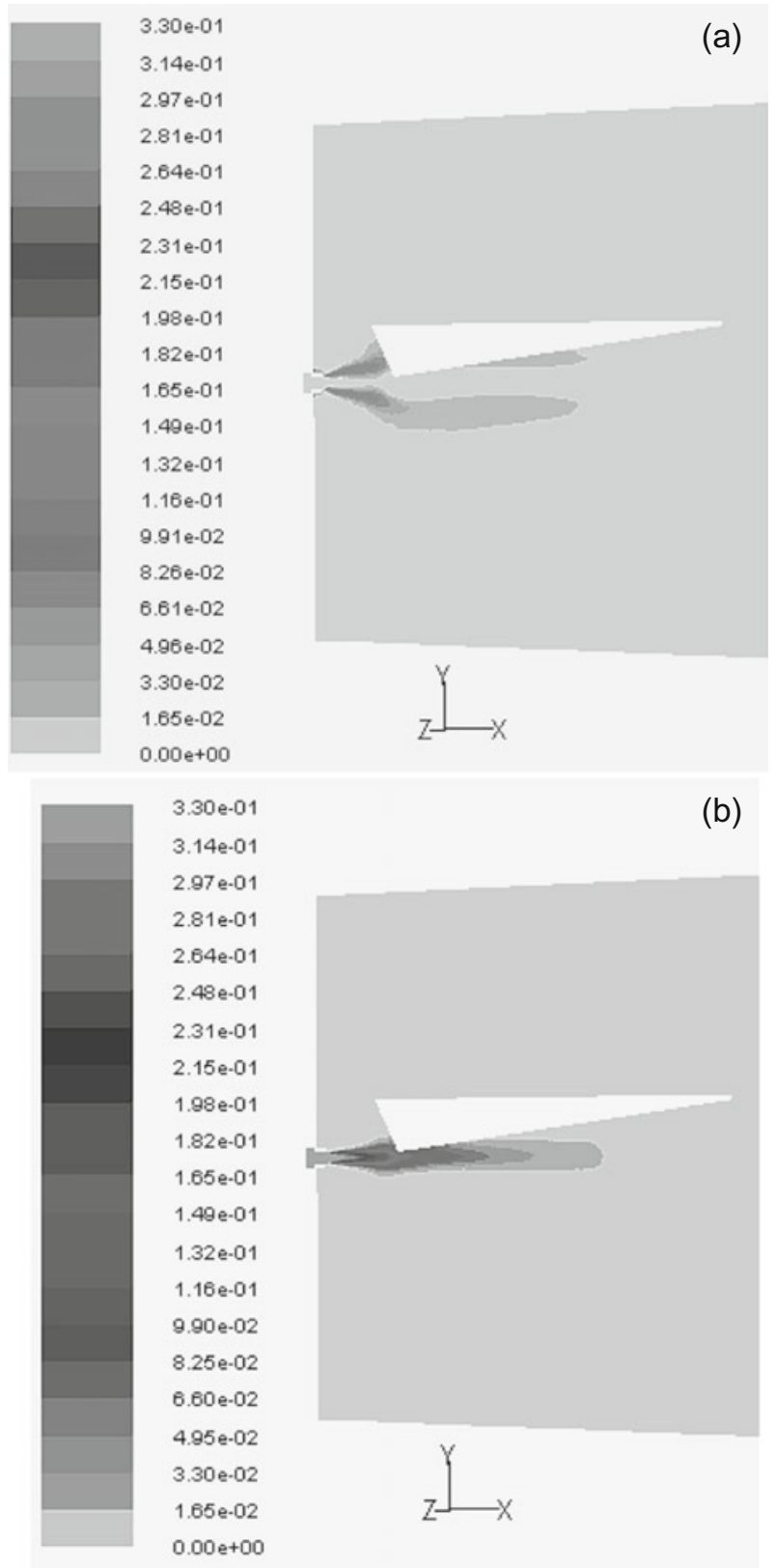

Fig. 7. The calculated distribution of methane mass fraction in the plane of symmetry (methane oxidation was considered). Methane and argon were introduced (a) through the central nozzle and (b) through the outer one.

A heat cooler was placed behind the reactor to cool down the post-reaction gases. The samples of gases were collected from behind the cooler (Fig. 10). The quantitative composition of post-reaction gases was determined using two gas chromatographs Shimadzu 2014 equipped with TCD and FID detectors. The Supelco's chromatographic column of Carboxen-1000 ( $5 \mathrm{ft} \times 1 / 8$ " SS, 40/60 mesh) was applied to determine the concentration of $\mathrm{H}_{2}, \mathrm{O}_{2}, \mathrm{CO}$ and $\mathrm{CO}_{2}$ in post-reaction gases. Hydrocarbons in the postreaction gases were determined using the J\&W's column of GS-Carbonplot $(30 \mathrm{~m} \times 0.533 \mathrm{~mm} \times 3.00 \mu \mathrm{m})$. The
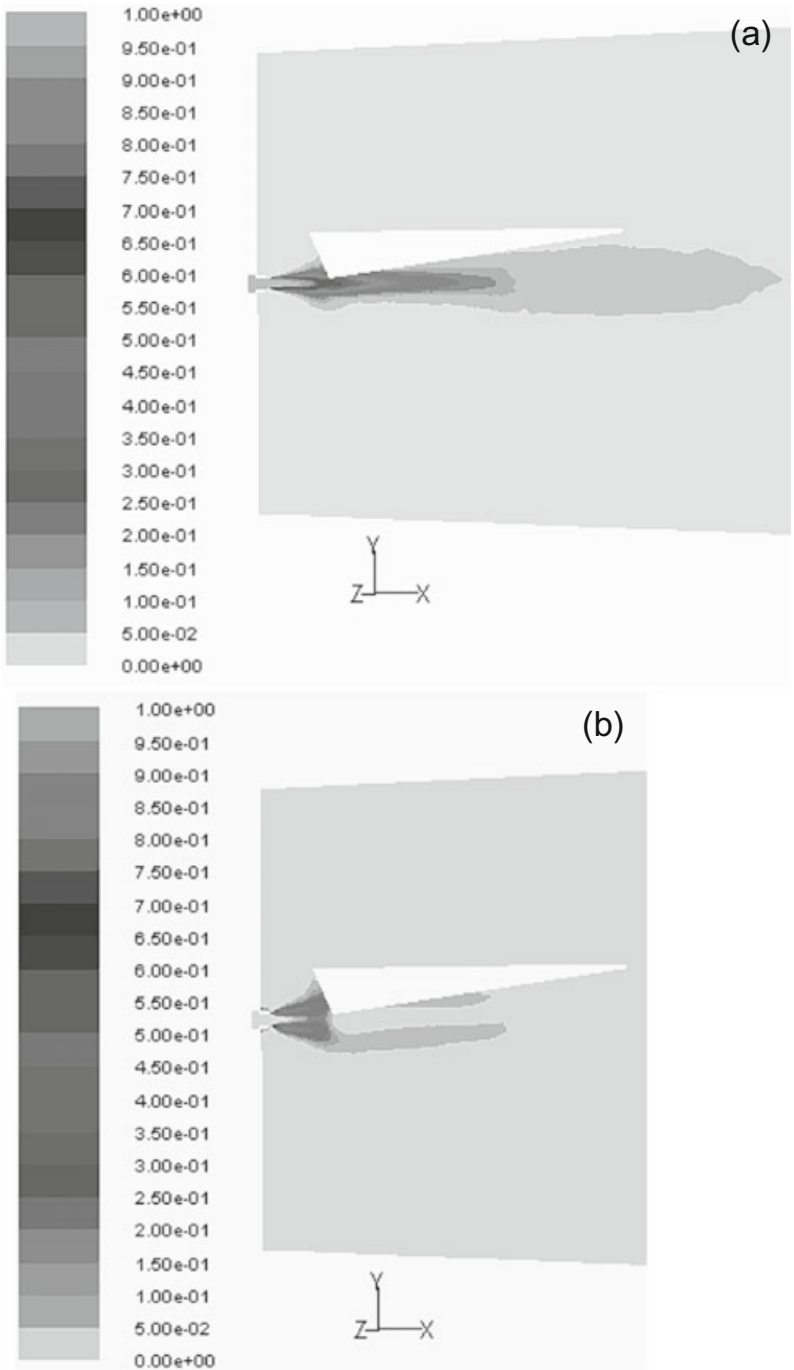

(b)

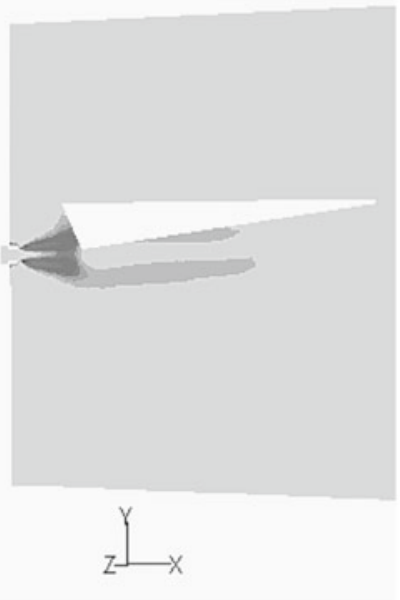

Fig. 8. The calculated distribution of oxygen mass fraction in the plane of symmetry (methane oxidation was considered). Methane and argon were introduced (a) through the central nozzle and (b) through the outer one.

concentration of components in post-reaction gases was indicated using an external standard method (the standard mixtures of Air Liquide and Messer).

The process parameters were defined as follows:

- The total conversion of methane $(X)$ :

$$
X=\left(V_{0 \mathrm{CH}_{4}}-V_{k \mathrm{CH}_{4}}\right) / V_{0 \mathrm{CH}_{4}},
$$

where: $V_{0 \mathrm{CH}_{4}}$ - methane flow at the reactor inlet $\left[\mathrm{Nm}^{3} / \mathrm{h}\right] ; V_{k \mathrm{CH}_{4}}-$ methane flow at the outlet of the heat exchanger $\left[\mathrm{Nm}^{3} / \mathrm{h}\right]$.

- The conversion of methane into carbon dioxide $\left(\mathrm{XCO}_{2}\right)$ :

$$
X \mathrm{CO}_{2}=V_{k \mathrm{CO}_{2}} / V_{\mathrm{OCH}_{4}},
$$

where: $V_{k \mathrm{CO}_{2}}$ - flow of carbon dioxide at the outlet of the heat exchanger $\left[\mathrm{Nm}^{3} / \mathrm{h}\right]$.

- The conversion of methane into carbon oxide (XCO):

$$
X \mathrm{CO}=V_{k \mathrm{CO}} / V_{0 \mathrm{CH}_{4}} .
$$



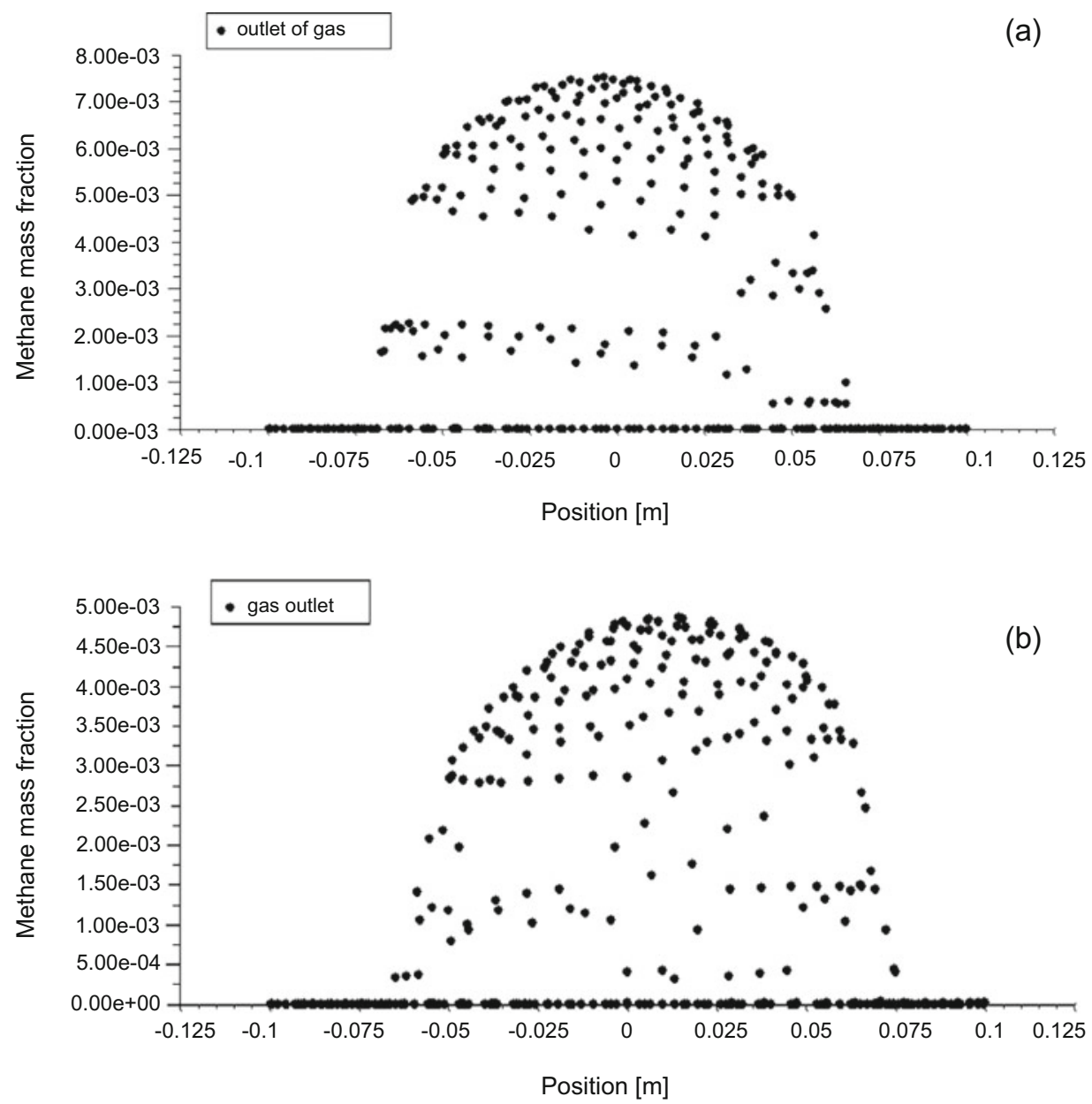

Fig. 9. The distribution of methane mass fraction at the outlet of reactor calculated numerically including the methane oxidation. Methane and argon were introduced (a) through the outer nozzle and (b) through the central mixing nozzle.

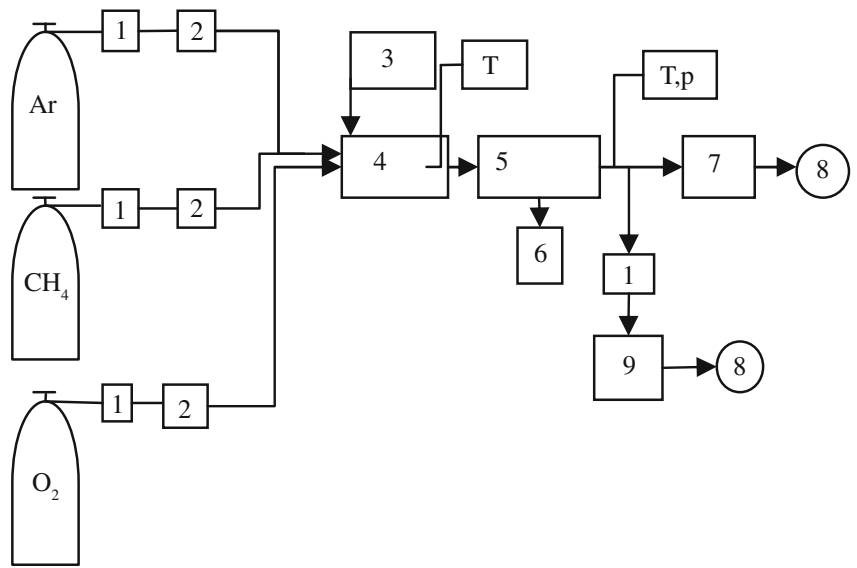

Fig. 10. The scheme of experimental setup: (1) an electromagnetic valve, (2) a mass flow meters, (3) a supply power, (4) a plasma reactor, (5) a gas cooler, (6) a water collector, (7) a gas meter, (8) exhaust, (9) a system for off-gas sampling, T, $p-\mathrm{a}$ temperature and pressure measurement.

- The specific energy $\left(E_{w}\right)$ :

$$
E_{w}=P \times 3.6 \times \frac{22.4}{V_{\text {in }}}[\mathrm{kJ} / \mathrm{mol}]
$$

where: $P$ - power $[\mathrm{kW}], V_{\text {in }}$ - the total inlet flow rate (the sum of flow rates of argon, methane and oxygen at the reactor inlet $\left.\left[\mathrm{Nm}^{3} / \mathrm{h}\right]\right) ; 22.4\left[\mathrm{Ndm}^{3} / \mathrm{mol}\right]$ the volume of one mol of ideal gas in normal conditions.

- The energy consumption $(Y)$ :

$$
Y=\frac{P \times 3.6 \times 22.4}{V_{0 \mathrm{CH}_{4}} \times X} \times 10^{-3}[\mathrm{~kJ} / \mathrm{mol}] .
$$

- The ratio of flow rates of gas flowing through the central nozzle and that through the outer one $(z)$ :

$$
z=\frac{V_{0 \mathrm{CH}_{4}}+V_{0 \mathrm{Ar}}}{V_{0 \mathrm{O}_{2}}},
$$

where: $V_{0 \mathrm{Ar}}, V_{0 \mathrm{O}_{2}}$ - argon and oxygen flow rates at the inlet of reactor $\left[\mathrm{Nm}^{3} / \mathrm{h}\right]$, respectively.

\subsection{Results}

Experiments were made for three inlet total gas flow rates $3.45,5.38$ and $7.50 \mathrm{Nm}^{3} / \mathrm{h}$ for each of the two methods of the gas introduction into the reactor with the aim of 
T. Opalińska et al.: Numerical simulation and experimental verification of gas streams

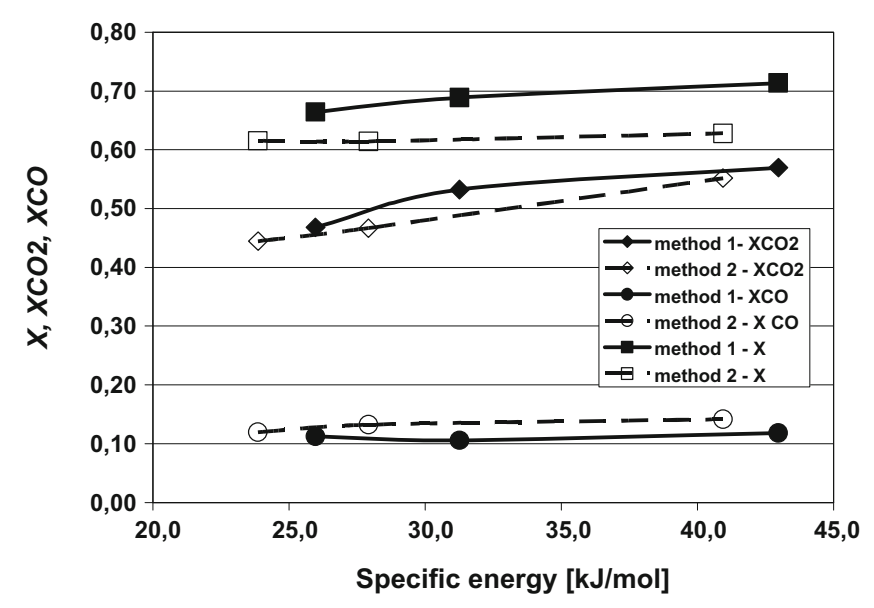

Fig. 11. The methane conversion vs. the specific energy for two methods of introduction of methane and argon mixture to the reactor: (1) through the mixing nozzle; (2) through the outer nozzle. The initial concentration of methane in the input gases was $3.15 \pm 0.09$ vol.\% and the sum of flow rates of gases feeding the reactor was $3.45 \div 7.50 \mathrm{Nm}^{3} / \mathrm{h}$.

testing the proper functioning of the system in a wide range of gas flow rates. The supplied power increased with the flow rate and it changed in the range of $1.7 \div 2.4 \mathrm{~kW}$. For this reason, the specific energy was used as the main parameter. The methane concentration in the total inlet flow was $3.15 \pm 0.09 \mathrm{vol} \%$. This means that through the central nozzle was flowing 3.06, 4.88 and $6.70 \mathrm{Nm}^{3} / \mathrm{h}$ of methane and argon mixture. The flow rate through the outer nozzle was: $0.37,0.59$ and $0.82 \mathrm{Nm}^{3} / \mathrm{h}$. The ratio of flow rates of gas flowing through the central nozzle and that through the outer one was constant and equal to $8.25 \pm 0.11$. The oxygen to methane molar flow rates were $3.46 \pm 0.06$. The results of experiments are presented in Figure 11.

They confirmed the conclusion drawn on the basis of calculations. The first method of gas introduction into the reactor was better than the second one. When gases were introduced by the first method, $X$ and $X \mathrm{CO}_{2}$ were higher and $X \mathrm{CO}$ was lower than the respective parameters for the gases introduced by the second method. The influence of the method of gases introduction into the reactor on the final process parameters (methane conversion) increased with the total gas flow. This conclusion corresponds well with the results of numerical simulations of gas streams feeding the reactor which were made for the total gas flow of up to $10 \mathrm{Nm}^{3} / \mathrm{h}$.

On the other hand, it can be supposed that the differences in methane conversion obtained in experiments arise from the significant disparity between the gas flow rates through particular nozzles. It can inhibit the stream mixing and blending in the whole volume of the stream. In the experiments the stream flow through the central nozzle was about eight times higher than that of the outer nozzle. Therefore, not only the method of gases introduction into the reactor can influence the process of mixing, but also the ratio of gas flow rate through the nozzles. This hypothesis was verified by additional experiments,

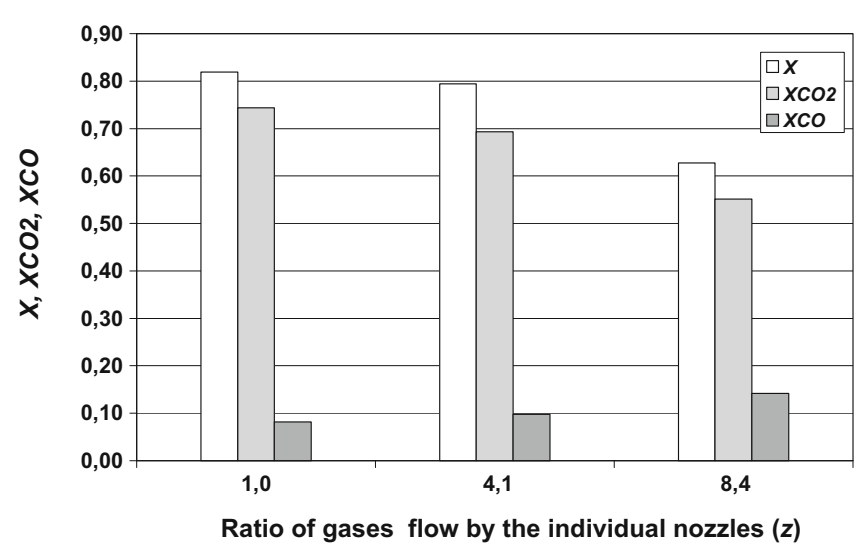

Fig. 12. The total methane conversion and the conversion of methane to $\mathrm{CO}_{2}$ and $\mathrm{CO}$ vs. the ratio of gas flow by the individual nozzles. The total gas flow rate was $3.48 \pm 0.02 \mathrm{Nm}^{3} / \mathrm{h}$. The methane concentration in the total initial flow of gases was $3.01 \pm 0.01$ vol.\%.

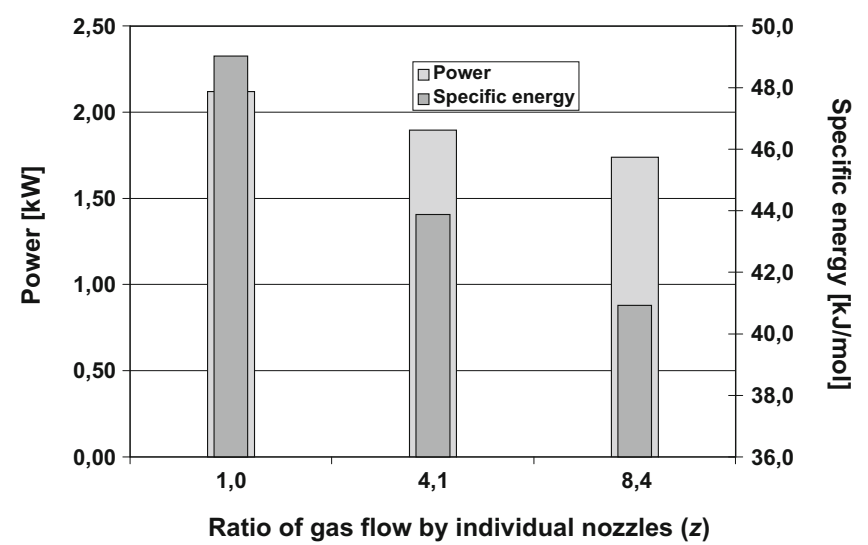

Fig. 13. The changes of the power, the specific energy and the energy consumption vs. the ratio of gas flow by the individual nozzles. The total gas flow rate was $3.48 \pm 0.02 \mathrm{Nm}^{3} / \mathrm{h}$. The methane concentration in the total initial flow of gases was $3.01 \pm 0.01$ vol. $\%$.

in which the first method of gases introducing into the reactor was used.

The experiments were made for the total gas flow equal to $3.48 \pm 0.02 \mathrm{Nm}^{3} / \mathrm{h}$. The ratios of flow rate of gases fed to the reactor through the central nozzle to that fed through the outer nozzle were 1.0 and 4.1. The ratio was changed by the addition of argon to oxygen stream. The methane concentration in the total initial flow of gases was $3.01 \pm 0.01$ vol. $\%$. The results of the experiments are shown in Figures 12 and 13. They were compared with the data from Figure 11 obtained for the total flow rate of gases $3.45 \mathrm{Nm}^{3} / \mathrm{h}$ and for $(z)=8.4$.

The $X, X_{2} \mathrm{CO}_{2}$ and $X \mathrm{CO}$ obtained for $(z)=8.4$ differ significantly from those obtained for $z=4.1$. Whereas the differences between the methane conversion obtained for $(z)=4.1$ and 1.0 did not vary substantially. It can be expected that the process conditions would be the best when $z \geq 4$.0. However, it is necessary to take into account the 
energy parameters (Fig. 13). The power decreased with a rise of $(z)$. It was caused by the changes of argon and oxygen concentrations in the total flow of gases fed to the reactor. The ratio of argon to oxygen flow rate was 8.1 for $(z)$ equal to 8.4 and 7.2 for $(z)$ equal to 4.1 and 1.0 . Then the specific energy decreased with a drop of power for a constant total flow rate of gases. It can cause that methane conversion decreases for rising $(z)$. It can be concluded that the methane conversion depended not only on the $(z)$ ratio but also on the input energy and thus on the specific energy. In the numerical simulation, $(z)$ was about 1.0. The best experimental methane conversions were obtained for the same value of $(z)$. Therefore we can assume that for a given construction of nozzles the effects of the chemical reaction depend not only on the method of gas feeding, but also on the ratio of flow rates through the outer and inner nozzles.

\section{Summaries}

The application of plasma reactor to the oxidation of hydrocarbons in the device for the waste disposal required the theoretical work-out and the experimental verification of the methods of gas feeding by means of two nozzles. The calculations made with the CFC Fluent program led to the conclusion that the most effective oxidation of hydrocarbons in the plasma reactor takes place when the stream of argon and hydrocarbons is introduced into the reactor through the central nozzle and oxygen through the outer one. Methane was used as a test hydrocarbon. The results of experiments confirmed the correctness of conclusion drawn from the calculations and show that the ratio of flow rates of gases through the internal nozzle and outer one is the important parameter. The best methane conversions are obtained for the ratio equal to 1.0, which was considered in theoretical calculations.

This work was supported by The National Centre for Research Development and carried out within the frame of project N R14 0023 06/2009.

\section{References}

1. Ch.S. Kalra, A.F. Gustol, A.A. Fridman, IEEE Trans. Plasma Sci. 33, 32 (2005)

2. A.A. Fridman, J. Chapelle, A. Czernichowski, L. Lesueur, P. Petrusev, B. Potapkin, J. Stevefelt, in Proceedings of the 11th International Symposium on Plasma Chemistry, edited by J.E. Harry (International Organising Committee, Loughborough, 1993), p. 415

3. K. Krawczyk, B. Ulejczyk, Plasma Chem. Plasma Process. 23, 265 (2003)

4. http://www.glidarc-tech.com

5. T. Opalińska, A. Opalska, K. Schmidt-Szałowski, Plasma Processes and Polymers (WILEY-VCH Verlag GmbH \& Co. KGaA, Weinheim, 2005), p. 415

6. T. Zieliński, T. Opalińska, J. Kijeński, Plasma Processes and Polymers (WILEY-VCH Verlag GmbH \& Co. KGaA, Weinheim, 2005), p. 443

7. E. Kowalska, T. Opalińska, J. Radomska, B. Ulejczyk, Vacuum 82, 1069 (2008)

8. A. Caramel, A. Czernichowski, A. Gorius, Patent US 5711859

9. A. Czernichowski, P. Czernichowski, Environ. Protect. Eng. 36, 37 (2010)

10. A. Czernichowski, K. Krawczyk, T. Opalińska, K. SchmidtSzałowski, J. Setek, Patent PL 196319

11. P. Czernichowski, A. Czernichowski, Patent WO 00/13786

12. A. Fridman, A. Chirokov, A. Gutsol, J. Phys. D: Appl. Phys. 38, R1 (2005)

13. J. Piechna, W. Selerowicz, T. Opalińska, B. Ulejczyk, M. Kalczewska, in Proceedings of the 7th International Conference ELMECO-7, Electromagnetic Devices and Process in Environment Protection, Natęczów, 2011, p. 127

14. J. Piechna, W. Selerowicz, T. Opalińska, B. Ulejczyk, M. Kalczewska, in Proceedings of the 7th International Conference ELMECO-7, Electromagnetic Devices and Process in Environment Protection, Nateczów, 2011, p. 125

Open Access This article is distributed under the terms of the Creative Commons Attribution Noncommercial License which permits any noncommercial use, distribution, and reproduction in any medium, provided the original author(s) and source are credited. 